\title{
Electrodynamics of a substorm-related field line resonance observed by the Polar satellite in comparison with ground Pi2 pulsations
}

\author{
A. Keiling, ${ }^{1}$ K.-H. Kim, J. R. Wygant, and C. Cattell \\ School of Physics and Astronomy, University of Minnesota, Minneapolis, Minnesota, USA \\ C. T. Russell \\ Institute of Geophysics and Planetary Physics, University of California, Los Angeles, Los Angeles, California, USA \\ C. A. Kletzing \\ Department of Physics and Astronomy, University of Iowa, Iowa City, Iowa, USA
}

Received 29 December 2001; revised 24 February 2003; accepted 2 April 2003; published 8 July 2003.

[1] Observations from the Polar satellite during a substorm on 29 August 1996 are used to characterize electrodynamic properties of a nightside field line resonance (FLR). A negative $H$ bay and Pi2 pulsations were simultaneously observed at midlatitude SAMNET ground stations $(L=2.6-4.5, \sim 0000 \mathrm{MLT})$. During the pulsations, Polar was moving outbound on a 0330 MLT meridian and crossing $L$ shells from 4 to 5.3. While in the central plasma sheet and outside the plasmasphere, Polar recorded toroidal standing waves for $\sim 20$ min starting simultaneously with the ground pulsations. The magnetic-fieldaligned Poynting flux associated with the FLR was $\leq 4 \mu \mathrm{W} \mathrm{m}{ }^{-2}$. Whereas on the ground at $L \leq 4$, the Pi2 frequency remained nearly constant and was similar for all stations, the toroidal frequency of the in situ FLR $(L \geq 4)$ decreased as the satellite crossed field lines with increasing $L$ value. Although this $L$ dependence of frequency of in situ FLR has been inferred in previous studies using multiple satellites at different local times, the 29 August 1996 event directly confirms this frequency dependence for substorm-related FLR over a large $L$ range on the same meridian. Furthermore, ground $(L \leq 4)$ and satellite $(L \geq 4)$ observations allow for the possibility that the oscillations were manifestations of two different magnetospheric oscillation modes, resulting from the same initial magnetospheric disturbance. INDEX TERMS: 2788 Magnetospheric Physics: Storms and substorms; 2764 Magnetospheric Physics: Plasma sheet; 2731 Magnetospheric Physics: Magnetosphere—outer; 2730 Magnetospheric Physics: Magnetosphere—inner; KEYWORDS: field line resonance, Pi2 pulsations, substorm, Poynting flux, electrodynamics, magnetospheric oscillation modes

Citation: Keiling, A., K.-H. Kim, J. R. Wygant, C. Cattell, C. T. Russell, and C. A. Kletzing, Electrodynamics of a substorm-related field line resonance observed by the Polar satellite in comparison with ground Pi2 pulsations, J. Geophys. Res., 108(A7), 1275, doi:10.1029/2002JA009340, 2003.

\section{Introduction}

[2] The characterization of field line resonance (FLR) in the magnetosphere is an ongoing effort in space physics. Although the theoretical description of the elementary FLR is well established [e.g., Southwood, 1974], the magnetospheric FLR phenomenon is not understood to full satisfaction. With the launch of new satellites, carrying more complete instrumentation than previous satellites, and having new orbits, thus probing different regions in space, we can increase our understanding of FLR. The Polar satellite allows to take a new look at substorm-related FLR.

\footnotetext{
${ }^{1}$ Now at Centre d'Etude Spatiale des Rayonnements, Toulouse, France.
}

Copyright 2003 by the American Geophysical Union. 0148-0227/03/2002JA009340\$09.00
[3] Previous studies of FLR can be divided into those focusing on dayside events [Zanetti et al., 1987; Singer et al., 1982; Cahill et al., 1986; Lin et al., 1992], on nightside events [Hughes and Grard, 1984; Takahashi et al., 1996; Kim et al., 2001], those occurring simultaneously with substorms [e.g., Takahashi et al., 1988; Nosé et al., 1998; Keiling et al., 2001b], and those triggered by a northward turning of IMF [Laakso et al., 1998]. The focus of this study was to determine the electrodynamics of a nightside FLR that was initiated at substorm onset.

[4] The Polar satellite has a highly elliptical, polar orbit with apogee and perigee of $\sim 9 R_{E}$ and $\sim 1.8 R_{E}$, respectively. During the early years of its operation, Polar's orbit crossed $L$ shells in the range from 3 to 10 in the Northern Hemisphere in $\sim 1$ hour, making it suitable to investigate the $L$ dependence of FLR which can last for $\sim 10-20 \mathrm{~min}$ 
[Takahashi et al., 1996]. We show the $L$ dependence of the frequency of substorm-related FLR during one satellite crossing of a range of $L$ shells. Previous studies of substorm-related FLR inferred their $L$ dependence from simultaneous measurements of a number of satellites that were located at different local times and different $L$ values [Takahashi et al., 1996; Kim et al., 2001].

[5] In addition, the Polar instrumentation provides the opportunity to investigate the electrodynamics of these substorm-related FLR. Previous studies, investigating $L$ dependent FLR in the nightside, either only had magnetic field data available [Kim et al., 2001] or used calculations of ion moments to infer the electric field [Takahashi et al., 1996] to establish their standing wave nature. Measurements of the electric field and magnetic field using the UC Berkeley Electric Field Instrument [Harvey et al., 1995] and the UCLA FLuxgate Magnetometer [Russell et al., 1995] on Polar directly confirm that the waves are standing. Furthermore, we show calculations of Poynting flux in association with these FLR. Using data from the particle detector Hydra of the University of Iowa [Scudder et al., 1995] and the electric field instrument, we show that the FLR occurred outside the plasmasphere and inside the central plasma sheet (CPS). Preliminary results of a statistical study of 24 FLR events also place these events outside the plasmasphere.

[6] Low and midlatitude ground stations from the SubAuroral Magnetometer Network (SAMNET) are used to confirm that the satellite observations of FLR were made during a typical substorm. Furthermore, the ground Pi2s occurring inside the plasmasphere show a different frequency dependence compared to the in situ FLR.

\section{Plasma Sheet Pass on 29 August 1996}

[7] Figure 1 presents the plasma sheet crossing by Polar on 29 August 1996. The top panel shows the $E_{x}$ component of the electric field. The second panel shows the eastward perturbation of the magnetic field $B_{y}$. The field data are presented throughout this paper in a field-aligned coordinate system in which the $z$ axis is parallel to the local magnetic field, $\mathrm{B}$, the $x$ axis is perpendicular to $\mathrm{B}$ and points away from the Earth in the meridional plane, and the $y$ axis is perpendicular to $\mathrm{B}$ and points in an easterly direction. The next two panels show the plasma density inferred from the spacecraft potential [Pedersen, 1995] and the Hydra particle detector. The last panel shows the energy-time spectrogram of electrons from Hydra.

[8] Polar was moving outbound from the plasmasphere into the plasma sheet near the 0330 MLT meridian (along a trajectory east of the SAMNET array, 0000 MLT). Polar crossed the plasmapause at about 2315 UT, shortly after which it entered the central plasma sheet (CPS), identified by the energy dispersion in the electron data. Shortly thereafter, FLR oscillations were recorded in the electric and magnetic fields. These oscillations lasted until 2339 UT when Polar encountered a large-amplitude electric field pulse $\left(E_{x} \sim 100 \mathrm{mV} / \mathrm{m}\right)$. This marked the beginning of a variety of physical changes: $B_{y}$ increased sharply by $\sim 60 \mathrm{nT}$, Polar left a region of enhanced electron energy flux and entered a region of enhanced ion energy flux (not shown), and the tail magnetic field dipolarized as determined from the other two magnetic field components (not shown).
Although these observations are interesting in their own right, we will not further discuss them here but focus on the FLR oscillations. The reader is referred to the works of Keiling et al. [2001a] and Toivanen et al. [2001] in which large field fluctuations during substorms are further discussed.

[9] Figures $2 \mathrm{a}$ and $2 \mathrm{~b}$ show expanded views of electric and magnetic field components which are perpendicular to each other. Both components were band-pass filtered between 10 and $120 \mathrm{~s}$. The same field-aligned coordinate system is used as in Figure 1. Figure 2c shows the $E_{x}$ component overlaid on the Hilbert-transformed magnetic field component, $H\left\{B_{y}\right\}$. Since the Hilbert transform of a signal shifts all Fourier components by $90^{\circ}$, the fact that both components are in phase confirms that these pulsations are standing Alfven waves, i.e., FLR. It can also be seen that the frequency decreased as Polar moved to larger $L$. We will further comment on this frequency change below. The last panel shows the magnetic field-aligned Poynting flux calculated from the two components shown in this figure. The Poynting flux oscillates with varying amplitudes up to $4 \mu \mathrm{W} \mathrm{m}{ }^{-2}$.

[10] This event occurred during an isolated substorm which was preceded by about 4 hours of weak geomagnetic activity. Figure 3, which plots the $H$ component of the magnetic field at Oulu (OUL) and $B_{y}$ from Polar, shows a negative deflection in the $H$ component starting at 2321 UT which we identify as the onset time of the substorm. Magnetic field oscillations in the Pi2 frequency range began simultaneously both on the ground and at Polar. At 2333 UT the substorm electrojet was reduced for a short period of time (seen in the excursion of $H$ ), after which it again intensified at $\sim 2335$ UT. The disturbances at OUL also showed strong negative vertical excursions in the $z$ component (not presented) indicating that the electrojet was centered to the south of this station. The oscillations at Polar lasted longer than those recorded on the ground and their intensity increased until 2339 UT when $B_{y}$ sharply increased, indicating the occurrence of a strong field aligned current.

[11] Figure 4 shows the band-pass filtered (10 s, $100 \mathrm{~s}) H$ component from all SAMNET stations that had data available and the three magnetic field components at Polar. The time interval corresponds to the $\mathrm{Pi} 2$ pulsation associated with the substorm expansion phase. Although the ground stations were located at different $L$ values ( $L=2.57$ to 4.48$)$, they all recorded pulsations with approximately the same frequency among themselves. In addition, a phase reversal occurred between GML and OUL ground stations. This is a typical substorm ground signature as reported by many authors [e.g., Lester and Orr, 1983; Yeoman and Orr, 1989]. We also note that OUL showed the largest amplitudes and a second intensification at $\sim 2330$ UT which was not recorded by the lower $L$ value ground stations.

[12] The in situ oscillations of the three magnetic field components show simularities and differences among themselves. $B_{z}$ and $B_{y}$ started out with similar waveforms and frequencies except for a phase shift of $\sim 90^{\circ}$. After about 2324 UT, $B_{y}$ decreased in amplitude but continued to show clear oscillations which later on increased in amplitude. The oscillations in $B_{z}$ also weakened after 2324 UT but only show clear oscillations again after 2330 UT. Although less sinusoidal, the $B_{z}$ oscillations show similar frequencies 


\section{Plasma sheet pass on 29 August 1996}

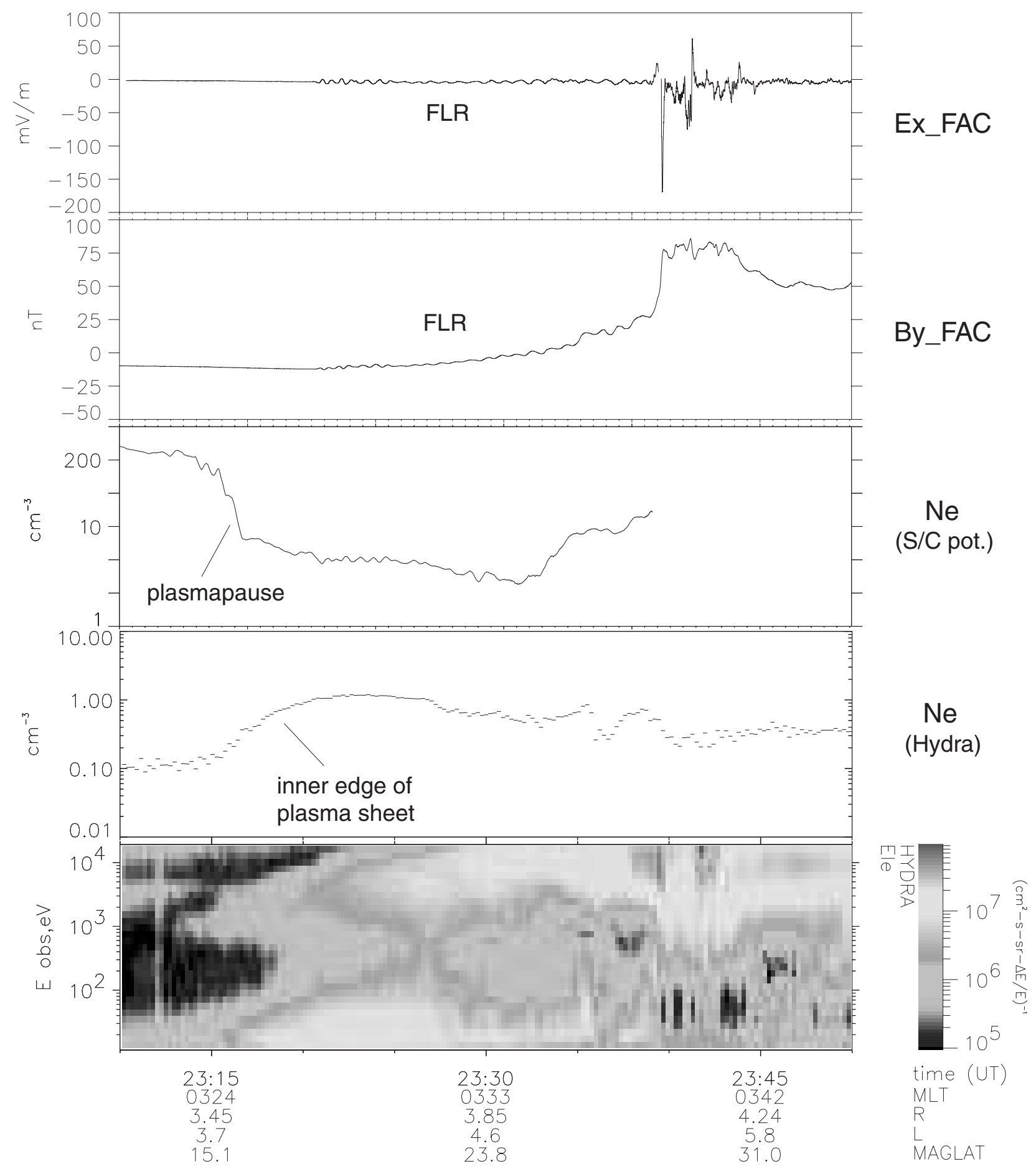

Figure 1. Measurements from the Polar satellite on 29 August 1996, during a plasma sheet crossing. The panels show, from top to bottom, the radial electric field component normal to the background magnetic field, the east-west component of the magnetic field (model subtracted), electron density determined from the spacecraft potential, electron density determined from the Hydra particle detector, and energy-time spectrogram of electrons. See color version of this figure at back of this issue.

compared to $B_{y}$ after 2333 UT. The magnitude of $B_{z}$ was about $30 \%$ compared with that of $B_{y}$ until 2324 UT, after which it was $\leq 15 \%$. Whereas $B_{y}$ and $B_{z}$ had the same onset time, clear oscillations in $B_{x}$ only started about 1 min later.
The $B_{x}$ oscillations were less sinusoidal than $B_{y}$. In the following the focus is on $B_{y}$.

[13] As was shown earlier, $B_{y}$ is associated with FLR. The fourth panel shows explicitly the frequency variation of $B_{y}$ 


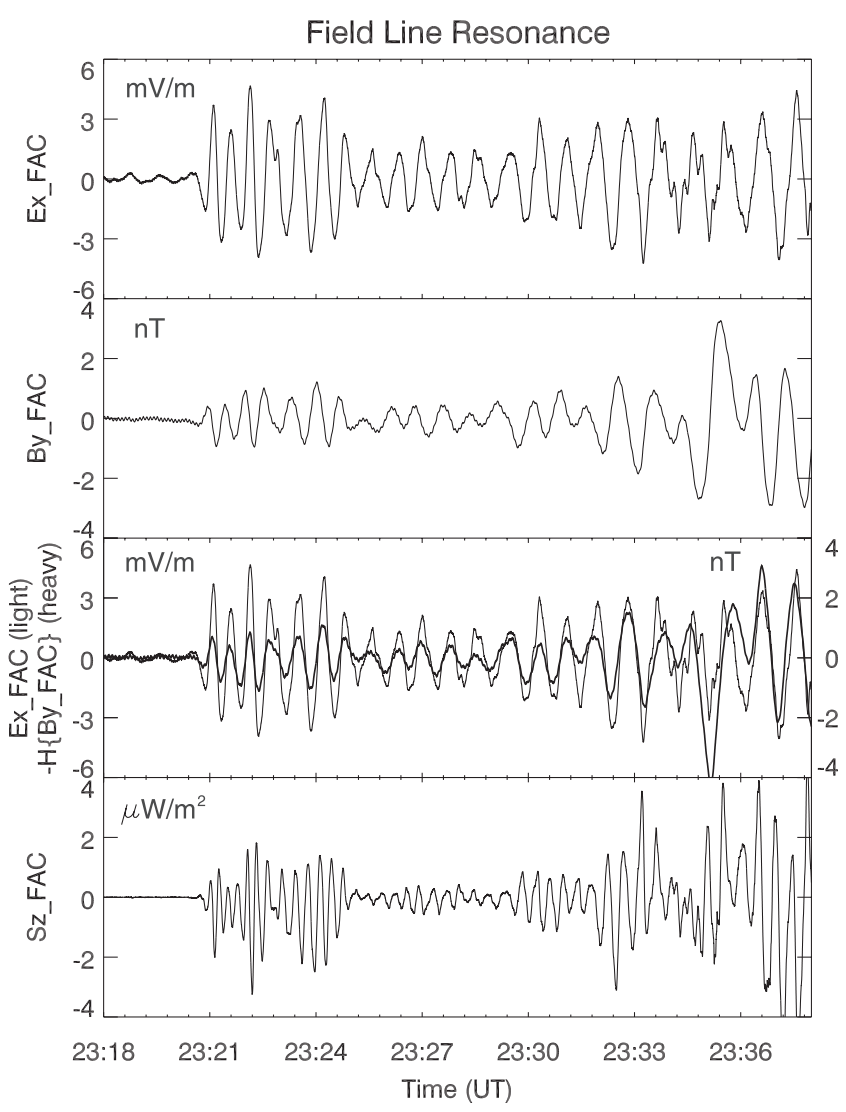

Figure 2. Field data for the FLR observed by Polar on 29 August 1996. (a) $E_{x}$ component of the electric field. (b) $B_{y}$ component of the magnetic field. (c) $E_{x}$ and the Hilberttransformed $B_{y}$ overlaid. (d) The Poynting flux associated with the two field components. Data were band-pass filtered $(10 \mathrm{~s}, 120 \mathrm{~s})$. These data show the standing wave nature and the frequency variation of the pulsations.

as a function of $L$ value. The solid line is the $L$ value crossed by Polar. The dots show the frequency of $B_{y}$ determined from counting the time period between two wave crests into frequency. The frequency decreases with increasing $L$ value as expected from a simple FLR model of the magnetosphere. The spread of the dots about a best-fit line (not drawn) is partially due to phase skips in the $B_{y}$ component indicated by arrows in the third panel. If field lines with different $L$ value oscillate independently with different frequencies, one might expect complicated phase relationships between neighboring field lines. This phase relationship becomes a function of time and $L$ value. The phase skips in $B_{y}$ might be indicators of this effect. In spite of the error in the frequency values introduced due to the phase skips, there is a clear trend in the data towards lower frequency for larger $L$ values. These satellite observations are in agreement with a toroidal resonance of the magnetosphere excited by a broadband frequency source.

[14] In addition to the single event, we surveyed Polar orbits of 1997 (nightside only) in a preliminary study, resulting in about 490 plasma sheet passes. Polar's polar orbit precesses throughout the year, thus covering all magnetic local times with uniform data coverage. Events were visually identified on the basis of oscillation in $B_{y}$ (azimuthal component) which stood out from the background. Twenty four events were identified and their locations as a function of magnetic local time versus dipole $L$ value is plotted in Figure 5. A concentration of FLR events in the postmidnight sector can be seen. For each event, electric and magnetic fields and plasma density data were investigated. It was found that for all events $E_{z}$ and $B_{y}$ were $90^{\circ}$ out of phase, which indicates their standing wave nature. The azimuthal component dominated over the compressional component. Furthermore, the density data shows that all FLR events occurred outside the plasmasphere and inside the plasma sheet similar to the 29 August 1996 event.

\section{Summary and Discussion}

[15] The focus of this study was to determine some electrodynamic properties of a nightside FLR (29 August $1996)$ in space ( $\sim 330$ MLT, $\left.L \sim 4-5.3,3.6-4 R_{E}\right)$ which was initiated at substorm onset and to compare the space observations with simultaneous ground Pi2 observations. In situ electric and magnetic field measurements by the Polar satellite confirmed their standing wave nature. Poynting flux calculations yielded values up to $4 \mu \mathrm{W} \mathrm{m} \mathrm{m}^{-2}$. For a single satellite pass we reported the $L$ dependence of the FLR frequency, decreasing from 35 to $15 \mathrm{mHz}$ while the satellite crossed $L$ shells from 4 to 5.3 during a time period of $20 \mathrm{~min}$. These observations confirm previous results of substorm-related, $L$-dependent FLR derived on the basis of multisatellite studies but show for the first time direct measurements of the electric fields and associated Poynting flux of this type of substorm-related FLR. Takahashi et al. [1996] showed that the frequencies of FLR were different at different local times and different $L$ shells, using three

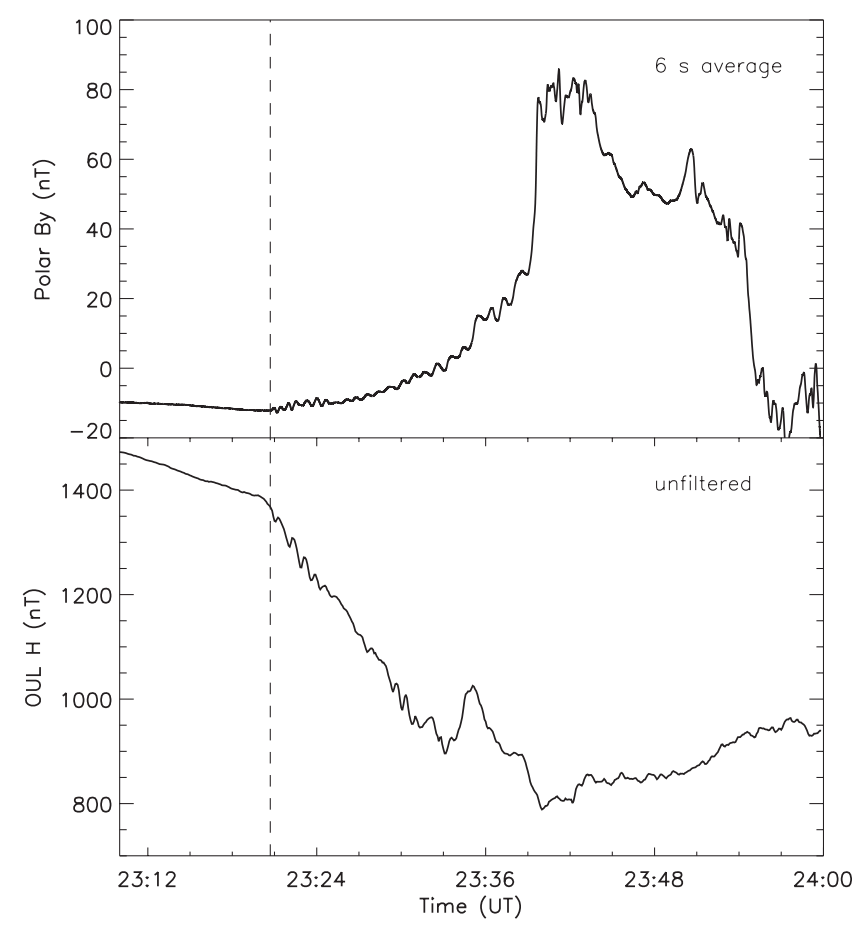

Figure 3. Magnetic field measurements from (a) Polar and (b) the ground station Oulu (OUL). The data show that pulsations started simultaneously in space and on the ground. 

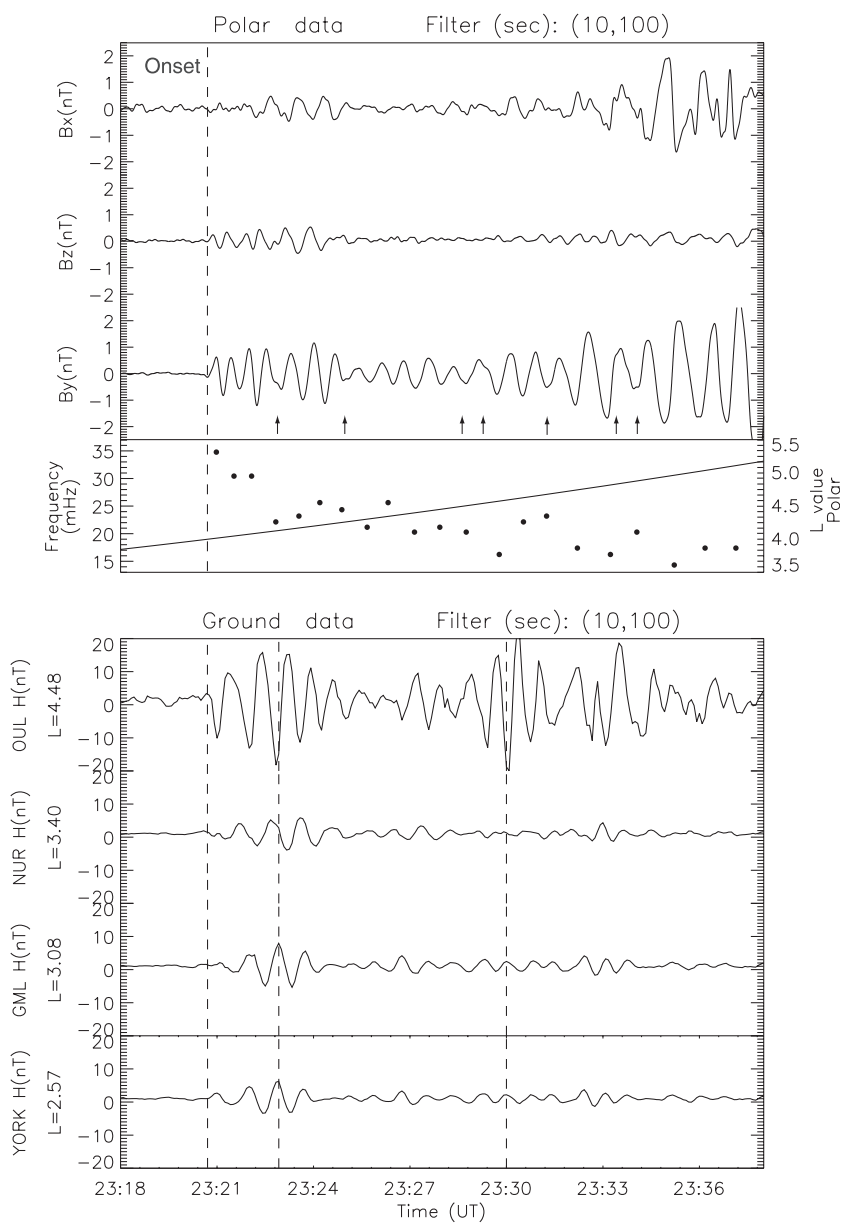

Figure 4. Comparison of waveforms in space and on the ground on 29 August 1996. The data were band-pass filtered $(10 \mathrm{~s}, 100 \mathrm{~s})$. The first three panels show the radial $\left(B_{x}\right)$, the field-aligned $\left(B_{z}\right)$ and the azimuthal $\left(B_{y}\right)$ component of the magnetic field as recorded by Polar. The fourth panel shows the $L$ value (solid line) crossed by Polar together with the frequency variation of $B_{y}$ (dots) throughout the crossing of the plasma sheet. Arrows indicate phase skips. The last four panels show the $H$ component of SAMNET ground stations.

satellites close to the equatorial plane. The FLR reported here was observed at larger magnetic latitude $\left(\geq 19^{\circ}\right)$.

[16] While Polar recorded FLR, a negative $H$ bay and Pi2 pulsations were observed at midlatitude SAMNET ground stations $(L=2.6-4.5, \sim 0000$ MLT). In spite of the spatially largely separated observing sites, the onset of the space oscillations coincided with the onset of the ground $\mathrm{Pi} 2 \mathrm{~s}$. This suggests that the space FLR and ground Pi2s resulted from the same initial magnetospheric disturbance. It is somewhat surprising that the transit times from the $\mathrm{Pi} 2$ source to either of the two observing sites happen to be the same. On the other hand, it cannot be ruled out that the space FLR existed before the onset of ground Pi2s, and Polar was entering this FLR region exactly at the time of the ground $\mathrm{Pi} 2$ onset. This is, however, a very unlikely scenario. A statistical study is planned to further investigate whether the simultaneous onset time of space FLR and ground Pi2s was coincidental. We conclude that our observations are one additional piece of evidence that $L$-dependent FLR are generated at substorm onset in addition to ground Pi2s.

[17] Although the ground and space oscillations were observed at the same time, they show important differences. Whereas the ground Pi2s showed the same frequency over a wide range of $L$ values $(L \leq 4)$, the in situ FLR $(L \geq 4)$ showed $L$-dependent frequencies. Polar showed the location of the plasmapause at $L=3.7$, thus placing the in situ FLR outside the plasmasphere. Particle data showed that the FLR occurred inside the plasma sheet. Because the SAMNET ground stations were located at $\sim 0000$ MLT and it is known that the plasmapause location varies with magnetic local time, in particular, it extends further out in the premidnight to midnight region, we cannot determine whether all ground stations were inside the plasmasphere. It is however most likely that NUR, GML, and YOR $(L=2.57-3.4)$ were conjugate to plasmaspheric field lines. These stations recorded nearly identical Pi2 oscillations. Global oscillations in the plasmasphere at a single frequency have been interpreted by some authors as cavity-type modes which are standing compressional magnetohydrodynamic waves bouncing between an inner and an outer boundary [e.g., Yeoman and Orr, 1989; Takahashi et al., 1995; Lee, 1998; Cheng et al., 2000].

[18] The space FLR, on the other hand, could not have been part of a possible cavity oscillation. A satellite crossing field lines belonging to the cavity should detect oscillations at a single frequency in the magnetic field component along the direction of the background magnetic field. Although Polar observed oscillations in all three magnetic field components, with $B_{y}$ being the dominant oscillation, the oscillations do not support a cavity-type mode interpretation at Polar's location. Instead the $L$-dependence of the FLR frequency is in agreement with a toroidal resonance in the

\section{Equatorial Projection of FLRs}

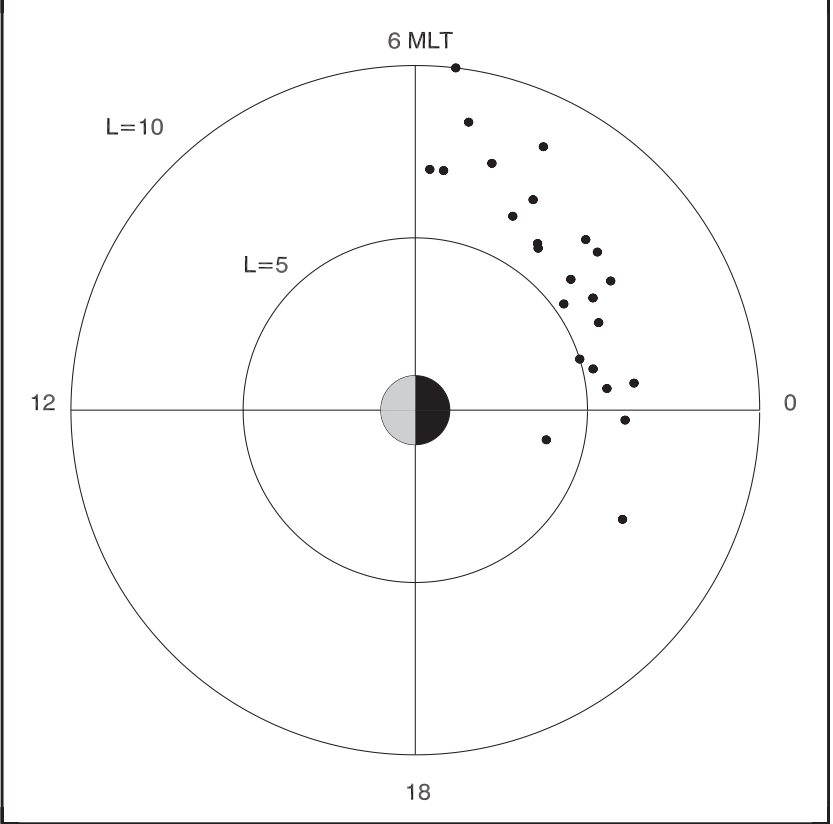

Figure 5. Equatorial projection of FLR events observed by Polar during 1997 . Note that only the nightside (18000600 MLT) was surveyed. 
plasma sheet excited by a broadband frequency source. It needs to be reemphasized, however, that Polar was not magnetically conjugate to the ground stations. In fact, for the most part, Polar crossed field lines with $L$ values larger than those that were conjugate to the SAMNET ground stations (with the exception of OUL) placing Polar outside the plasmasphere and most of the ground stations inside the plasmasphere. Thus we suggest that the oscillations inside the plasmasphere and in the plasma sheet were manifestations of two different magnetospheric oscillation modes, which is no contradiction because they occurred in different regions of space.

[19] In comparison, Keiling et al. [2001b] reported strong FLR in the plasma sheet at $\sim 2300$ MLT, which were also triggered at substorm onset, with a significant compressional component $B_{z}$. It was demonstrated that the FLR was mode-coupled to a fast mode wave. In their event the FLR did not show any $L$ dependence. Since the FLR had the same frequency as the ground $\mathrm{Pi} 2$ pulsations, a cavity-type mode feeding the FLR was considered a possible scenario by the authors. In spite of the significant differences in space observations of Keiling et al. [2001b] and the study presented here, both studies show similar ground pulsations. To understand the reason why the space signatures are different, one has to remember that the satellite observations of both studies were made at very different local times and different magnetic latitudes. It is known that $\mathrm{Pi} 2 \mathrm{~s}$ in space are mostly observed near local midnight [Takahashi et al., 1995], even if they are observed well around the Earth on the ground. Since our observations were made at 0330 MLT, it is tempting to think that a spacecraft, located at midnight and conjugate to the ground stations used in this study, would have observed a similar signature as reported by Keiling et al. [2001b] (observations of fast mode waves coupled to FLR). It is also possible that the larger MLAT $\left(\geq 19^{\circ}\right)$ in our study is partially responsible for a weak signal in $B_{z}$, because cavity-type mode oscillations are likely to concentrate near the equator in dipolar geometry [Takahashi et al., 1995].

[20] In an attempt to explain space and ground observations made by Lin et al. [1992], Rickard and Wright [1995] used a magnetospheric waveguide model. The study by Lin et al. [1992] looked at an FLR event in the dayside which was excited by solar wind variations. It was noted that the compressional and the azimuthal components were different. The compressional oscillations were less sinusoidal and lasted shorter than the FLR. Both the fast mode and the FLR showed $L$-dependent frequency variations. Rickard and Wright [1995] showed that these signatures could be simulated by assuming a waveguide model and a broadband source applied to the dayside magnetosphere, simulating solar wind variations. The simulation supported the view that the FLR and the fast mode waves were associated with an MHD waveguide. This interpretation could be applied to the 29 August 1996 event bearing in mind the differences, namely that this event occurred in the nightside dawn flank and was clearly substorm-related.

[21] The statistical study by Takahashi et al. [1996] recorded most FLR events in the postmidnight sector. The 29 August 1996 event lies in this region. Our preliminary results of a statistical study of nightside FLR, using the Polar data set, also show FLR events predominately in the postmidnight sector. In addition, the Polar data set shows that all events occurred outside the plasmasphere and inside the plasma sheet similar to the 29 August 1996 event. Events were found both very close to the inner edge of the plasma sheet and deeper inside the CPS. This shows that FLR can occur throughout the CPS. It also seems likely that FLR can simultaneously occupy the entire CPS. The 29 August 1996 event supports this view because the latitudinal extent, as recorded by Polar while crossing different $L$ shells, was only limited by the lifetime ( $\sim 17 \mathrm{~min})$ of the source as seen in the ground Pi2. Interestingly, the in situ FLR lasted somewhat longer than the ground Pi2s and also increased in intensity as time went on. The different duration times may be interpreted in terms of boundary differences. For the FLR the boundaries are the northern and southern ionospheres, but for the ground Pi2s (if they are associated with trapped fast mode waves), boundaries in the north-south, radial, and azimuthal directions are required to confine the wave energy. This means that the fast mode energy has more ways of leaking than FLR oscillations.

[22] Acknowledgments. Analysis of electric field data was supported by NASA International Solar Terrestrial Program (NASA contract NAG 5-3182). Analysis of magnetometer data was supported by NASA NAG 5-7721. Work at the University of Iowa in analysis of Hydra data was performed under NASA grant 5-2231 and DARA grant 50 OC 89110 . We thank D. Milling for the SAMNET magnetometer data. SAMNET is a PPARC National Facility deployed and operated by the University of York.

[23] Lou-Chuang Lee thanks Dong-Hun Lee and two other reviewers for their assistance in evaluating this paper.

\section{References}

Cahill, L. J., et al., Electric and magnetic observations of the structure of standing waves in the magnetosphere, J. Geophys. Res., 91, 8895, 1986. Cheng, C.-C., et al., Spectral power of low-latitude Pi2 pulsations at the 210 magnetic meridian stations and plasmaspheric cavity resonances, Earth Planets Space, 52, 615-627, 2000.

Harvey, P., et al., The electric field instrument on the Polar satellite, in The Global Geospace Mission, edited by C. T. Russell, Kluwer Acad., Norwell, Mass., 1995.

Hughes, W. J., and R. J. L. Grard, A second harmonic geomagnetic field line resonance at the inner edge of the plasma sheet: GEOS 1, ISEE 1, and ISEE 2 observations, J. Geophys. Res., 89, 2755, 1984.

Keiling, A., et al., Properties of large electric fields in the plasma sheet at 4-7 RE measured with Polar, J. Geophys. Res., 106, 5779, 2001a.

Keiling, A., et al., Pi2 pulsations observed with the Polar satellite and ground stations: Coupling of trapped and propagating fast mode waves to a midlatitude field line resonance, J. Geophys. Res., 106, 25,891, $2001 \mathrm{~b}$

Kim, K.-H., et al., A comparison of $\mathrm{Pi} 2$ pulsations in the inner magnetosphere and magnetic pulsations at geosynchronous orbit, J. Geophys. Res., 106, 18,865, 2001

Laakso, H., et al., Field-line resonances triggered by a northward IMF turning, Geophys. Res. Lett., 25, 2991, 1998.

Lee, D.-H., On the generation mechanism of $\mathrm{Pi} 2$ pulsations in the magnetosphere, Geophys. Res. Lett., 25, 583, 1998.

Lester, M., and D. Orr, Correlation between ground observations of Pi2 geomagnetic pulsations and satellite plasma density observations, Planet. Space Sci., 31, 143, 1983.

Lin, N., et al., Field and thermal plasma observations of ULF pulsations during a magnetically disturbed interval, J. Geophys. Res., 97, 14,859, 1992.

Nosé, M., et al., ULF pulsations observed by the ETS-VI satellite: Substorm associated azimuthal Pc4 pulsations on the nightside, Earth Planet. Space, 50, 63, 1998.

Pedersen, A., Solar wind and magnetosphere plasma diagnostics by spacecraft electrostatic potential measurements, Ann. Geophys., 13, 118, 1995.

Rickard, G. J., and A. N. Wright, ULF pulsations in a magnetospheric waveguide: Comparison of real and simulated satellite data, J. Geophys. Res., 100, 3531, 1995.

Russell, C. T., et al., The GGS/Polar magnetic fields investigation, in The Global Geospace Mission, p. 563, edited by C. T. Russell, Kluwer Acad., Norwell, Mass., 1995. 
Scudder, J., et al., Hydra-A 3-dimensional electron and ion hot plasma instrument for the Polar spacecraft of the GGS mission, in The Global Geospace Mission, p. 495, edited by C. T. Russell, Kluwer Acad., Norwell, Mass., 1995.

Singer, H. J., W. J. Hughes, and C. T. Russell, Standing hydromagnetic waves observed by ISEE 1 and 2: Radial extent and harmonic, J. Geophys. Res., 87, 3519, 1982.

Southwood, D. J., Some features of field line resonances in the magnetosphere, Planet. Space Sci., 22, 483, 1974.

Takahashi, K., et al., AMPTE/CCE observations of substorm-associated standing Alfven waves in the midnight sector, Geophys. Res. Lett., 15, 1287, 1988 .

Takahashi, K., et al., Statistical analysis of Pi2 pulsations observed by the AMPTE CCE spacecraft in the inner magnetosphere, J. Geophys. Res., 100, 21,929, 1995.

Takahashi, K., et al., Multisatellite study of nightside transient toroidal waves, J. Geophys. Res., 101, 24,815, 1996.

Toivanen, P. K., et al., Plasma sheet dynamics observed by the Polar spacecraft in association with substorm onsets, J. Geophys. Res., 106, 19,117, 2001.
Yeoman, T. K., and D. Orr, Phase and spectral power of mid-latitude Pi2 pulsations: Evidence for a plasmaspheric cavity resonance, Planet. Space Sci., 37, 1367, 1989.

Zanetti, L. J., et al., Geomagnetic field-line resonant harmonics measured by the Viking and AMPTE/CCE magnetic field experiments, J. Geophys. Res., 14, 427, 1987.

C. Cattell, A. Keiling, K.-H. Kim, and J. R. Wygant, School of Physics and Astronomy, University of Minnesota, 116 Church Street S, Minneapolis, MN 55455, USA. (cattell@belka.space.umn.edu; akeiling@ham. space.umn.edu; khan@belka.umn.edu; wygant@belka.umn.edu)

C. A. Kletzing, Department of Physics and Astronomy, University of Iowa, Iowa City, IA 52242, USA. (craig-kletzing@uiowa.edu)

C. T. Russell, Institute of Geophysics and Planetary Physics (IGPP), University of California, Los Angeles, Los Angeles, CA 90095, USA. (ctrussel@igpp.ucla.edu) 


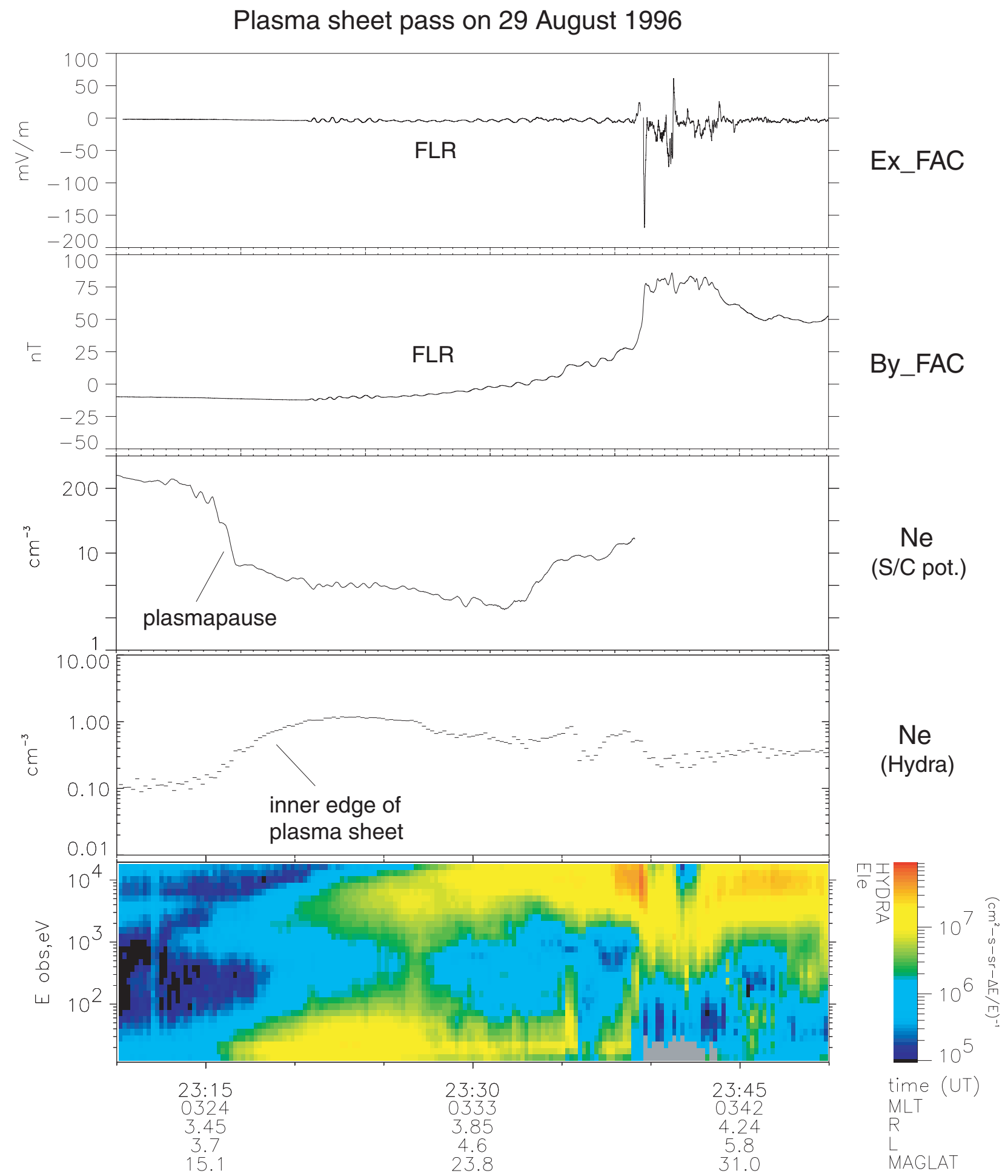

Figure 1. Measurements from the Polar satellite on 29 August 1996, during a plasma sheet crossing. The panels show, from top to bottom, the radial electric field component normal to the background magnetic field, the east-west component of the magnetic field (model subtracted), electron density determined from the spacecraft potential, electron density determined from the Hydra particle detector, and energy-time spectrogram of electrons. 